\title{
LOCAL MARTINGALES WITH TWO REFLECTING BARRIERS
}

\author{
MATS PIHLSGÅRD, ${ }^{*}$ Lund University
}

\begin{abstract}
We give an account of the characteristics that result from reflecting a drifting local martingale (i.e. the sum of a local martingale and a multiple of its quadratic variation process) in 0 and $b>0$. We present conditions which guarantee the existence of finite moments of what is required to keep the reflected process within its boundaries. Also, we derive an associated law of large numbers and a central limit theorem which apply when the input is continuous. Similar results for integrals of the paths of the reflected process are also presented. These results are in close agreement to what has previously been shown for Brownian motion.
\end{abstract}

Keywords: Skorokhod problem; reflection; stochastic integration; Brownian motion; local martingale; semimartingale

2010 Mathematics Subject Classification: Primary 60G44; 60G51; 60H05

Secondary $60 \mathrm{G} 17$

\section{Introduction}

In recent years many authors have studied problems which can be characterized as reflections of some input. There have been a large variety of problems investigated with different reflection schemes (one-sided reflection, two-sided reflection, reflection at current maximum, etc.) and types of input (e.g. Lévy processes and semimartingales). We are deliberately vague since we want to use the phrases 'input', 'feeding process', etc. to denote a deterministic function as well as a random process. The same rule applies to 'reflected process', 'local times', etc. Throughout this paper we will assume that we are given a real-valued càdlàg input $X=$ $(X(t), t \geq 0)$ with $X(0)=0$. As an appetizer we provide a few references together with short descriptions (this reference list is by no means complete) which contain treatments of different reflection-type problems under various assumptions about the input $X:$ [1] reflected Lévy processes, [2] reflected Lévy processes, [3] reflected Lévy processes with emphasis on the reflection mechanism itself, [6] reflected Lévy processes with an emphasis on the case where the Lévy measure is light-tailed, [8] reflection of a Markov modulated Brownian motion, [11] on fundamental issues concerning reflection, [12] reflection of Lévy process with a functional, i.e. not constant, upper barrier, [13] discrete time reflection, [15] reflection of Lévy process in a functional upper barrier, [16] discrete time reflection, and [21] discrete time reflection.

Perhaps the simplest reflection we can think of is when we force an input $X$ to stay above 0 . This is an example of a so-called Skorokhod problem. We wish to find $V$ with $V(t) \geq 0$ for $t \geq 0$ and $L$ with $L(0)=0$ which is nondecreasing, finite for all $t \geq 0$, and right-continuous

Received 25 April 2014; revision received 5 November 2014.

* Postal address: Clinical Research Centre, Bd. 28, Fl. 13, Jan Valdenströms gata 35, 20502 Malmö, Sweden.

Email address: mats.pihlsgard@med.lu.se 
(thus càdlàg) such that

$$
V(t)=V(0)+X(t)+L(t), \quad \int_{0}^{\infty} V(s) \mathrm{d} L(s)=0
$$

The integral condition in (1) says that $L$ increases only when $V$ is 0 . It is well known that there is an explicit solution to this problem given by

$$
L(t)=\left(-\inf _{0 \leq v \leq t} X(v)-V(0)\right)^{+}
$$

where $x^{+}=\max (0, x)$. Starting from (2) we can give conditions (typically restrictions on $X$ ) which, e.g. guarantee finite moments of $V$ and $L$ (see Theorem 1 below) and the existence of a stationary or asymptotic distribution of $V$.

Now, let us describe the two-sided reflection mechanism. It is (just like the one-sided reflection) a well-known (and well-described) Skorokhod problem. Given an input and a constant $b>0$, find $(V, L, U)$ with $0 \leq V(t) \leq b$ for $t \geq 0$ and $L, U$ satisfying $L(0)=$ $U(0)=0, L(t), U(t)$ nondecreasing, finite for all $t \geq 0$ and right-continuous such that

$$
V(t)=V(0)+X(t)+L(t)-U(t)
$$

and

$$
\int_{0}^{\infty} V(s) \mathrm{d} L(s)=0, \quad \int_{0}^{\infty}(b-V(s)) \mathrm{d} U(s)=0 .
$$

The process $V$ may be thought of as the process which results from reflecting $X$ in 0 and $b$, and $L$ and $U$ as the local times at 0 and $b$, i.e. the 'pushing away' from 0 and $b$, respectively. The conditions in (4), of course, say that $L$ and $U$ can increase only when $V$ is at the respective barrier. Pihlsgård and Glynn [17] contains a discussion about the existence and uniqueness of a solution $(V, L, U)$.

We stress that the Skorokhod problems described above are formulated for a deterministic input, i.e. they are defined path by path.

One main question is, of course, if it is possible to obtain an explicit formula for $V$ (containing $X$ and $b$ ) when we have two reflecting barriers. A partial answer in the affirmative to this question was given in [7]. In that paper a formula for $V$ was given which is valid when $X$ is of bounded variation. Rather recently, in Kruk et al. [14], an expression which applies to the general case was presented. We present it here because it is an impressive result which confirms that the solution to the two-sided reflection is, as expected, much more involved than that given in (2).

Proposition 1. Let $X^{\prime}(t)=V(0)+X(t)$. Then

$$
V(t)=X^{\prime}(t)-\left((V(0)-b)^{+} \wedge \inf _{u \in[0, t]} X^{\prime}(u)\right) \vee \sup _{s \in[0, t]}\left(\left(X^{\prime}(s)-b\right) \wedge \inf _{u \in[s, t]} X^{\prime}(u)\right)
$$

By inspecting (5) we observe that, strictly speaking, we are not given a complete solution to the Skorokhod problem since we are not able to identify $L$ and $U$. In view of (3), what we see is $L-U$. One idea is to try to identify $L$ and $U$ by constructing a decomposition of the signed measure corresponding to $X^{\prime}(t)-V(t)$ (see, e.g. [10, pp. 25-28] for a discussion concerning this issue) such that (4) holds. Note that the decomposition is not unique unless we impose the boundary conditions. However, this approach appears to be difficult. In [17] a different path based on (formal) integration of (3) was chosen. The conditions in (4) enter 
the calculations very conveniently. Since we will make frequent use of it, we state the main result from [17]. As always, if $f$ is a real-valued function then $\Delta f(s)=f(s)-f(s-)$, where $f(s-)=\lim _{u \uparrow s} f(u)$ (provided that the limit exists) with the convention that $f(0-)=0$. For a stochastic process $Y$, we let $[Y, Y]$ denote its quadratic variation process.

Proposition 2. Let

$$
\varphi(x, y)= \begin{cases}-\left(x^{2}+2 x y\right) & \text { if } y \leq-x \\ y^{2} & \text { if }-x<y<b-x, \\ 2 y(b-x)-(b-x)^{2} & \text { if } y \geq b-x\end{cases}
$$

Then

$$
U(t)=\frac{V(0)^{2}-V(t)^{2}+2 \int_{0+}^{t} V(s-) \mathrm{d} X(s)+[X, X]^{\mathrm{c}}(t)+J_{R}(t)}{2 b}
$$

where

$$
J_{R}(t)=\sum_{0<s \leq t} \varphi(V(s-), \Delta X(s))
$$

and $[X, X]^{\mathrm{c}}$ denotes the continuous part of $[X, X]$.

In [17] the authors pay the price of having to consider integrals where the 'differential' stems from a function of possibly infinite variation. Thus, in general the integral in (6) lacks Lebesgue-Stieltjes path-by-path significance. This obstacle is circumvented by letting integrals be of Itô type and taking $X$ to be a semimartingale.

In a sense, this paper starts off where [17] ends. We will narrow the perspective slightly and go from letting the input be a general semimartingale to considering local martingales with drift, i.e. $X(t)=M(t)+\mu[M, M](t)$, where $M$ is a local martingale and $\mu \in \mathbb{R}$. One motivation to study such objects is the following. A well-studied process is Brownian motion with drift, i.e. $X(t)=\sigma B(t)+\mu t, \mu \in \mathbb{R}, \sigma>0$, where $B$ is a standard Brownian motion. Of course, when we view $X$ as a Lévy process, we may think of it as the most general example with continuous sample paths, but if we decide to view it as a semimartingale, we insist that we should look at it as a scaled standard Brownian motion to which we add a constant multiplied by its quadratic variation process (which happens to be deterministic). Furthermore, the Dambis- DubinsSchwarz theorem (which tells us that a continuous local martingale can be represented as a time-changed Brownian motion; see [19, p. 181]) strongly suggests that it could be worthwhile to try to show results for drifting local martingales similar to that shown for Brownian motion with drift if we use a different scale to measure the drift.

The paper is organized as follows. In Section 2 we show a few results concerning moments of the local time $U$. Also, we include Proposition 3, which provides a representation of the local time $U$ (valid under certain conditions), and (as a slight digression) Proposition 4, which fills a minor gap in [17]. In Section 3 we present a few asymptotic results for $U$ and $V$, including laws of large numbers and central limit theorems for $U$ as well as for certain process obtained as an integral of the paths of $V$, which apply when the input is taken to be a continuous local martingale. This will further illustrate the connection between reflected Brownian motion and reflected local martingales.

\section{Moments and a result on the structure of $U$}

In this section we will systematically investigate moments of the local times $L$ and $U$ in (3) and (4). Since the details are very similar we will treat only $U$, but it should be obvious how 
to do the calculations for $L$. As a warm-up exercise we will have a short look at the one-sided reflection defined in (1) (with the solution given in (2)). Note that we are not able to prove the results in Theorem 1 for local martingales without imposing further conditions.

Theorem 1. Let $p \geq 1$. Assume without loss of generality that $V(0)=0$.

(i) If $X$ is a Lévy process then $\mathbb{E}\left[L^{p}(t)\right]<\infty$ if and only if $\mathbb{E}\left[X(1)^{p}\right]<\infty$.

(ii) If $X$ is a martingale such that $X(0)=0$, we have $\mathbb{E}[L(t)]<\infty$ if

$$
\sup _{0 \leq s \leq t} \mathbb{E}\left[X(s) \log ^{+} X(s)\right]<\infty
$$

and for $p>1 \mathbb{E}\left[L^{p}(t)\right]<\infty$ if and only if $\mathbb{E}\left[X(t)^{p}\right]<\infty$. Here $\log ^{+} x=$ $\max (0, \log |x|)$.

Proof. From (2) it follows that if $V(0)=0$ then $L(t)=\sup _{0 \leq s \leq t}(-X(s))$. Theorem 1(i) follows directly from [20, Theorem 25.18]. Theorem 1(ii) follows, for $p=1$, from a remark in $[19, \mathrm{p} .55]$ and for $p>1$ from Doob's inequality.

Theorem 1 tells us that the local time $L$ in (1) behaves more or less as expected as long as $X$ is a Lévy process or a martingale. However, when we consider a two-sided reflection the calculations become more involved. As we will see below, we require the finite $2 p$ th moment of $X^{*}$ in order to guarantee the finite $p$ th moment of $U(t)$, e.g. if $X$ is a martingale. For the case where $X$ is a Lévy process, it was proven in [6] that $\mathbb{E}[X(t)]<\infty$ yields $\mathbb{E}[U(t)<] \infty$ and in [17] that $\mathbb{E}\left[X^{2}(t)\right]<\infty$ implies $\mathbb{E}\left[U^{2}(t]\right)<\infty$, but we do not see an immediate way of generalizing these results to, e.g. the case where $X$ is a local martingale. Let us very briefly explain why the Lévy process case is easier than the case where the input is a local martingale. First, the stationary independent increments of a Lévy process leads to the reflected process being regenerative. This means that $U(t)$ can be split into independent contributions coming from different cycles and we can use powerful results from renewal theory when analyzing $U(t)$. Secondly, in the Lévy process case the continuous part can cause no problems as far as moments of $U(t)$ are concerned. Thirdly, we can control the intensities of small and large jumps via the Lévy measure (to the best of the author's knowledge there are no similar results which apply to martingales or local martingales) and this makes it possible to exploit the structure of $\varphi(\cdot, \cdot)$ (more precisely, for small $y, \varphi(x, y)$ is quadratic and for large $y$ it is linear) to obtain conditions guaranteeing the existence of finite moments of $J_{R}$ which are sharper than those presented below. We recall the Burkholder-Davis-Gundy inequalities, which make it possible to compare moments of $X$ and $[X, X]$ (when $X$ is a local martingale); see [18] and [19]. Thus,

$$
c_{p} \mathbb{E}[X, X]^{p / 2}(t) \leq \mathbb{E}\left[X^{*}\right]^{p}(t) \leq C_{p} \mathbb{E}[X, X]^{p / 2}(t), \quad p \in(0, \infty)
$$

when $X$ is continuous and for $p \geq 1$ when $X$ is càdlàg, where $c_{p}$ and $C_{p}$ are constants and $X^{*}(t)=\sup _{0<s \leq t}|X(s)|$.

Theorem 2. Let $p>0$. Assume without loss of generality that $V(0)=0$. If $X$ is a local martingale with drift generated by a continuous local martingale $M$ then, regardless of $\mu$, if $\mathbb{E}\left[M^{*}\right]^{2 p}(t)<\infty$ then $\mathbb{E}\left[U^{p}(t)\right]<\infty$.

Proof. From (6) it follows (recall that $X(t)=M(t)+\mu[M, M](t)$ ) that

$$
U(t)=\frac{V(0)^{2}-V(t)^{2}+2 \int_{0+}^{t} V(s) \mathrm{d} M(s)+2 \mu \int_{0+}^{t} V(s) \mathrm{d}[M, M](s)+[M, M](t)}{2 b}
$$


Clearly,

$$
\left|2 \mu \int_{0+}^{t} V(s) \mathrm{d}[M, M](s)+[M, M](t)\right| \leq(2|\mu| b+1)[M, M](t) .
$$

We have $Y(t)=\int_{0+}^{t} V(s) \mathrm{d} M(s)$ is a continuous local martingale such that

$$
[Y, Y](t)=\int_{0+}^{t} V(s)^{2} \mathrm{~d}[M, M](s) \leq b^{2}[M, M](t) .
$$

The claim now follows from

$$
\begin{aligned}
\mathbb{E}|Y(t)|^{p} & \leq \mathbb{E}\left[Y^{*}(t)\right]^{p} \\
& \leq C_{p} \mathbb{E}[Y, Y]^{p / 2}(t) \\
& \leq C_{p} b^{p} \mathbb{E}[M, M]^{p / 2}(t) \\
& \leq \frac{C_{p} b^{p} \mathbb{E}\left[M^{*}(t)\right]^{p}}{c_{p}} \\
& <\infty
\end{aligned}
$$

which holds in view of (7) and the assumption that $\mathbb{E}\left[M^{*}(t)\right]^{2 p}<\infty$.

We can provide a result similar to Theorem 2 for the case where $M$ is not continuous.

Theorem 3. Let $p \geq 1$. Assume without loss of generality that $V(0)=0$.

(i) If $\mu=0$ and $\mathbb{E}\left[M^{*}\right]^{2 p}(t)<\infty$, then $\mathbb{E}\left[U^{p}(t)\right]<\infty$.

(ii) If $\mu \neq 0$ and $\mathbb{E}\left[M^{*}\right]^{4 p}(t)<\infty$, then $\mathbb{E}\left[U^{p}(t)\right]<\infty$.

Proof. We start by noting that $\varphi(\cdot, \cdot)$ in Proposition 2 satisfies $0 \leq \varphi(x, y) \leq y^{2}$. Thus,

$$
[M, M]^{\mathrm{c}}(t)+\sum_{0<s \leq t} \varphi(V(s-), \Delta M(s)) \leq[M, M]^{\mathrm{c}}(t)+\sum_{0<s \leq t}(\Delta M(s))^{2}=[M, M](t)
$$

and Theorem 3(i) follows in the same way as Theorem 2. Now, if $X(t)=M(t)+\mu[M, M](t)$ then

$$
\begin{aligned}
J_{R}(t) & =\sum_{0<s \leq t} \varphi(V(s-), \Delta X(s)) \\
& =\sum_{0<s \leq t} \varphi\left(V(s-), \Delta M(s)+\mu(\Delta M(s))^{2}\right) \\
& \leq \sum_{0<s \leq t}\left(\Delta M(s)+\mu(\Delta M(s))^{2}\right)^{2} \\
& \leq c(2) \sum_{0<s \leq t}\left((\Delta M(s))^{2}+\mu^{2}(\Delta M(s))^{4}\right) \\
& \leq c(2)[M, M](t)+c(2) \mu^{2}[M, M]^{2}(t),
\end{aligned}
$$

where $c(2)$ is a finite constant. In the last step, if we let

$$
Y(t)=\left\{\sum_{0<s \leq t}(\Delta M(s))^{2}\right\}^{2}
$$

then $(\Delta M(t))^{4} \leq \Delta Y(t)$ and $Y(t) \leq[M, M]^{2}(t)$. Now, Theorem 3(ii) follows (again, in the same way as Theorem 2 ). 
When studying reflected Lévy processes, Brownian motion is by far the easiest example. In order to emphasize that its simple quadratic variation structure to a large extent accounts for its simplicity, we state the following simple corollary which applies to its local martingale counterpart (from a reflective perspective).

Corollary 1. Suppose that $X(t)=M(t)+\mu[M, M](t)$, where $[M, M]$ is deterministic and continuous (this is well known to imply that $M$ is a continuous martingale). Then all moments of $U(t)$ exist.

Proof. The corollary does not need a proof.

We conclude this section with two propositions which are intimately related to that presented in [17]. The first proposition summarizes the author's current understanding of the local time $U$ when the input is a martingale with finite second moment. It is an interesting problem to try to show that Proposition 3, or a result similar to it, holds under weaker assumptions. The second proposition is a slight digression and relates to reflection with a functional upper barrier $T$. Unfortunately, this result was overlooked in [17]. It makes it possible for us to sharpen [17, Lemma 1] (which leads to the important Theorem 3), where the assumptions are unnecessarily strict.

Proposition 3. Suppose that $X(t)=M(t)+\mu[M, M](t)$ with $\mu \geq 0$ and $\mathbb{E}\left[M^{2}(t)\right]<\infty$ (this means that $M$ is a martingale).

(i) If $M$ is continuous (or $\mu=0$ ) then $U$ has the representation

$$
U(t)=U_{1}(t)+U_{2}(t)
$$

where $-b / 2 \leq U_{1}(t) \leq b / 2$ and $U_{2}(t)$ is a submartingale.

(ii) If, in addition, $\mathbb{E}\left[M^{4}(t)\right]<\infty$ then (10) holds for discontinuous $M$ and $\mu>0$.

Proof. We identify $U_{1}(t)$ as $(2 b)^{-1}\left(V(0)^{2}-V(t)^{2}\right)$. Also, $\int_{0+}^{t} V(s-) \mathrm{d} M(s)$ is a local martingale such that its quadratic covariation process is given by $\int_{0+}^{t} V(s-)^{2} \mathrm{~d}[M, M](s)$ which is bounded by $b^{2}[M, M](t)$ and it follows by [18, Corollary 3] that $\int_{0+}^{t} V(s-) \mathrm{d} M(s)$ is a martingale. Propositions 3(i) and (ii) now follow easily from (6)- (9).

The two-sided reflection with functional upper barrier $T$ was described in [17], where it was assumed that $T$ is a semimartingale which fulfills $T(s) \geq \varepsilon>0$ for all $s \geq 0$ (with $\varepsilon$ a constant).

Proposition 4. Let $p>0$. If the input $X$ is a semimartingale such that $\mathbb{E}\left[U^{p}(t)\right]<\infty$ for constant upper barrier, then $\mathbb{E}\left[U^{p}(t)\right]<\infty$ for any functional barrier $T$ satisfying $T(s) \geq$ $\varepsilon>0$.

Proof. Assume without loss of generality that $V(0)=0$. Let $V_{T}, V_{\varepsilon}, L_{T}, L_{\varepsilon}, U_{T}$, and $U_{\varepsilon}$ denote the reflected processes and local times corresponding to reflection with $T$ and $\varepsilon$ as upper barrier, respectively. From the construction of $V_{T}$ given in [17, Proposition 1], in view of $T(s) \geq \varepsilon$, it follows that

$$
\left\{t \geq 0: V_{T}(t)=0\right\} \subseteq\left\{t \geq 0: V_{\varepsilon}(t)=0\right\}
$$


and, thus, $L_{T}(s) \leq L_{\varepsilon}(s)$. The claim now follows from

$$
\begin{aligned}
0 & \leq U_{T}(t) \\
& \leq U_{T}(t)+V_{T}(t) \\
& =X(t)+L_{T}(t) \\
& \leq X(t)+L_{\varepsilon}(t) \\
& =V_{\varepsilon}(t)+U_{\varepsilon}(t) \\
& \leq \varepsilon+U_{\varepsilon}(t) .
\end{aligned}
$$

\section{A few asymptotic results for a continuous input}

In this section we provide asymptotic results which apply when the input is continuous. We provide a law of large numbers and a central limit theorem for $U$. Furthermore, we obtain, more or less as a byproduct of the techniques used to show the aforementioned results, a corresponding result for certain integrals of the paths of $V$, which can be interpreted as time-averages. This shows yet another interesting connection between reflected Brownian motion (with drift) and reflected drifting local martingales. Zhang and Glynn [22] contains the corresponding investigation for reflected Brownian motion.

Recall the structure of the feeding process given by $X(t)=M(t)+\mu[M, M](t)$. In differential form (3) becomes

$$
\mathrm{d} V(t)=\mathrm{d} X(t)+\mathrm{d} L(t)-\mathrm{d} U(t)=\mathrm{d} M(t)+\mu \mathrm{d}[M, M](t)+\mathrm{d} L(t)-\mathrm{d} U(t) .
$$

Denote by $\mathcal{C}^{2}([0, b])$ functions from $[0, b]$ to $\mathbb{R}$ which are twice continuously differentiable. We take $f \in \mathcal{C}^{2}([0, b])$ and apply Itô's lemma and [17, Proposition 4] (which says that $[V, V](t)=$ $[X, X](t))$ to $(11)$, note that $[X, X](t)=[M, M](t)$, and thus obtain (we assume that $V(0)=0$ )

$$
\begin{aligned}
& f(V(t))-f(0) \\
&=\int_{0}^{t} f^{\prime}(V(s)) \mathrm{d} V(s)+\frac{1}{2} \int_{0}^{t} f^{\prime \prime}(V(s)) \mathrm{d}[V, V](s) \\
&= \int_{0}^{t} f^{\prime}(V(s)) \mathrm{d} M(s)+\mu \int_{0}^{t} f^{\prime}(V(s)) \mathrm{d}[M, M](s)+f^{\prime}(0) L(t) \\
&-f^{\prime}(b) U(t)+\frac{1}{2} \int_{0}^{t} f^{\prime \prime}(V(s)) \mathrm{d}[M, M](s) \\
&= \int_{0}^{t} f^{\prime}(V(s)) \mathrm{d} M(s)+f^{\prime}(0) L(t)-f^{\prime}(b) U(t) \\
&+\int_{0}^{t}\left\{\frac{1}{2} f^{\prime \prime}(V(s))+\mu f^{\prime}(V(s))\right\} \mathrm{d}[M, M](s) \\
&= \int_{0}^{t} f^{\prime}(V(s)) \mathrm{d} M(s)+f^{\prime}(0) L(t)-f^{\prime}(b) U(t)+\int_{0}^{t}(\mathscr{L} f)(V(s)) \mathrm{d}[M, M](s),
\end{aligned}
$$

where

$$
\mathcal{L}=\frac{1}{2} \frac{\mathrm{d}^{2}}{\mathrm{~d} x^{2}}+\mu \frac{\mathrm{d}}{\mathrm{d} x}
$$


Note that if we take $f(x)=x^{2}$, we arrive at (6). The idea of investigating the differential operator $\mathcal{L}$ comes from [22]. Example 1 below contains a short discussion about the differences between the techniques used in [22] and this paper.

In what follows we will assume that the only alternatives that occur are $[M, M](t) \rightarrow \infty$ almost surely (a.s.) and $[M, M](t) \rightarrow[M, M](\infty)$ a.s., respectively, as $t \rightarrow \infty$, for some finite random variable $[M, M](\infty)$. The reason is that we wish to avoid issues like state space decompositions, etc. Most of what remains of the paper is devoted to the first case. For the second case, we have the following result.

Proposition 5. Suppose that $[M, M](t) \rightarrow[M, M](\infty)<\infty$ a.s. as $t \rightarrow \infty$. Then $U(t) \rightarrow$ $U(\infty)<\infty$ a.s. and $V(t) \rightarrow V(\infty)$ a.s. If $\mathbb{E}[M, M](\infty)^{p}<\infty$ then $\mathbb{E}[U](\infty)^{p}<\infty$. Furthermore, $\mathbb{E}|V(t)-V(\infty)|^{p} \rightarrow 0$ and $\mathbb{E}|U(t)-U(\infty)|^{p} \rightarrow 0$ for all $p>0$.

Proof. Choose an injective $f \in \mathcal{C}^{2}([0, b])$ such that $f^{\prime}(0)=f^{\prime}(b)=0$. The part $\int_{0}^{t} f^{\prime}(V(s)) \mathrm{d} M(s)$ in (12) is a local martingale whose quadratic variation is bounded by $C^{2}[M, M](t)$, where $C=\sup _{s \in[0, b]} f^{\prime}(s)$. We now apply [19, Proposition 1.8] which says that for a continuous local martingale $M$,

$$
\left\{\lim _{t \rightarrow \infty}[M, M](t)<\infty\right\}=\left\{\lim _{t \rightarrow \infty} M(t) \text { exists }\right\}
$$

and we see that both terms in the rightmost part of (12) converge which means that $f(V(t))$ converges and so does $V(t)=\left(f^{-1} \circ f\right)(V(t))$. That $U(t)$ converges now follows if we apply the same arguments to (6). The rest follows by monotone convergence and dominated convergence in combination with the fact that $U$ is nondecreasing and $|V(t)| \leq b$.

Remark 1. When we derived (12) we used the fact that $[V, V](t)=[M, M](t)$. It could be tempting to try to show that this directly implies that $V(t) \rightarrow V(\infty)$ a.s. as $t \rightarrow \infty$. However, to the best of our understanding, we cannot conclude this since $V$ is a semimartingale, not a local martingale, and it does not follow automatically from (3) that $L(t)$ and $U(t)$ converge.

From now on we will assume that $[M, M](t) \rightarrow \infty$ a.s. as $t \rightarrow \infty$. For later use, we will now describe the solutions $f(\cdot)$ to the differential equations $(\mathcal{L} f)(x)=K+r(x)$ for a few $r$, where $K$ is a constant. It is fairly straightforward to show that

$$
f^{\prime}(x)=\mathrm{e}^{-2 \mu x} \int 2(K+r(x)) \mathrm{e}^{2 \mu x} \mathrm{~d} x
$$

This leads us to Table 1. The form of the solution is $f=f_{h}+f_{p}$, where $f_{h}$ solves the differential equation $\left(\mathcal{L} f_{h}\right)(x)=K$ and $f_{p}$ solves $\left(\mathscr{L} f_{p}\right)(x)=r(x)$. This means that the solution to $(\mathscr{L} f)(x)=K+c_{1} r^{1}(x)+\cdots+c_{n} r^{n}(x)$ is $f(x)=f_{h}(x)+c_{1} f_{p}^{1}(x)+\cdots+c_{n} f_{p}^{n}(x)$ (in the obvious notation), where $c_{1}, \ldots, c_{n}$ are constants. If $\mu=0$ then $f_{h}^{\prime}(x)=2 K x+K_{1}$ $\left(f_{h}(x)=K x^{2}+K_{1} x+K_{2}\right)$ and if $\mu \neq 0$ then $f_{h}^{\prime}(x)=K / \mu+K_{1} \mathrm{e}^{-2 \mu x}\left(f_{h}(x)=K x / \mu-\right.$ $K_{1} \mathrm{e}^{-2 \mu x} / 2 \mu+K_{2}$ ), where $K_{1}$ and $K_{2}$ are constants. Now, let us state and prove a law of large numbers for $U$.

Theorem 4. It holds that $U(t) /[M, M](t) \rightarrow c$ a.s. as $t \rightarrow \infty$, where

$$
c= \begin{cases}\frac{1}{2 b} & \text { if } \mu=0, \\ \frac{\mu}{\left(1-\mathrm{e}^{-2 \mu b}\right)} & \text { if } \mu \neq 0 .\end{cases}
$$


TABLE 1: Solutions to $\left(\mathcal{L} f_{p}\right)(x)=r(x)$.

\begin{tabular}{cccc}
\hline$\mu$ & $r(x)$ & $f_{p}^{\prime}(x)$ & $f_{p}(x)$ \\
\hline$=0$ & $x$ & $x^{2}$ & $x^{3} / 3$ \\
$=0$ & $x^{2}$ & $2 x^{3} / 3$ & $x^{4} / 6$ \\
$=0$ & $\mathrm{e}^{\alpha x}$ & $2 \mathrm{e}^{\alpha x} / \alpha$ & $2 \mathrm{e}^{\alpha x} / \alpha^{2}$ \\
$=0$ & $x \mathrm{e}^{\alpha x}$ & $2 \mathrm{e}^{\alpha x}(x-1 / \alpha) / \alpha$ & $2 \mathrm{e}^{\alpha x}(x-2 / \alpha) / \alpha^{2}$ \\
$\neq 0$ & $x$ & $-1 / 2 \mu^{2}+x / \mu$ & $-x / 2 \mu^{2}+x^{2} / 2 \mu$ \\
$\neq 0$ & $\mathrm{e}^{\alpha x}$ & $2 \mathrm{e}^{\alpha x} /(\alpha+2 \mu), \alpha \neq-2 \mu$ & $2 \mathrm{e}^{\alpha x} / \alpha(\alpha+2 \mu), \alpha \neq-2 \mu$ \\
$\neq 0$ & $\mathrm{e}^{\alpha x}$ & $2 x \mathrm{e}^{-2 \mu x}, \alpha=-2 \mu$ & $-\mathrm{e}^{-2 \mu x}(x+1 / 2 \mu) / \mu, \alpha=-2 \mu$ \\
\hline
\end{tabular}

Proof. Recall that we assume that $[M, M](t) \rightarrow \infty$ a.s. Let $f=f_{h}$, so that $(\mathcal{L} f)(x)=K$. Choose $K$ and $K_{1}$ such that $f^{\prime}(0)=0$ and $f^{\prime}(b)=1$. It turns out that $K=1 / 2 b$ and $K_{1}=0$ if $\mu=0$ and $K=\mu /\left(1-\mathrm{e}^{-2 \mu b}\right)$ and $K_{1}=-K / \mu$ if $\mu \neq 0$. In view of (12), we have

$$
U(t)=f(0)-f(V(t))+\int_{0}^{t} f^{\prime}(V(s)) \mathrm{d} M(s)+K[M, M](t)
$$

and, since $(f(0)-f(V(t))) /[M, M](t) \rightarrow 0$ as $t \rightarrow \infty$, it suffices to show that

$$
\int_{0}^{t} \frac{f^{\prime}(V(s)) \mathrm{d} M(s)}{[M, M](t)} \rightarrow 0 \text { a.s. }
$$

But $\int_{0}^{t} f^{\prime}(V(s)) \mathrm{d} M(s)$ is a local martingale with quadratic variation bounded by $C[M, M](t)$ for some constant $C$, which means that (14) follows directly from (13) and the law of large numbers for local martingales, which says that for a local martingale $Y, Y(t) /[Y, Y](t) \rightarrow 0$, $t \rightarrow \infty$ on $\{[Y, Y](t) \rightarrow \infty, t \rightarrow \infty\}$; see, e.g. [19, Exercise 1.16].

We will need the following lemma.

Lemma 1. Suppose that there exists a solution $f$ to the differential equation $(\mathcal{L} f)(x)=$ $K+r(x)$ such that $f^{\prime}(0)=f^{\prime}(b)=0$. Then it holds a.s. that

$$
\lim _{t \rightarrow \infty} \frac{1}{[M, M](t)} \int_{0}^{t} r(V(s)) \mathrm{d}[M, M](s)=-K .
$$

Proof. By (12), it follows that

$$
f(V(t))-f(0)=\int_{0}^{t} f^{\prime}(V(s)) \mathrm{d} M(s)+K[M, M](t)+\int_{0}^{t} r(V(s)) \mathrm{d}[M, M](s) .
$$

We divide both sides of (15) by $[M, M](t)$ and let $t \rightarrow \infty$. The claim follows by the law of large numbers for local martingales and $(f(0)-f(V(t))) /[M, M](t) \rightarrow 0$.

Theorem 5. Let $c$ be as in Theorem 4 and $M$ be a martingale with deterministic quadratic variation. As $t \rightarrow \infty$, it holds that

$$
\sqrt{[M, M](t)}\left(\frac{U(t)}{[M, M](t)}-c\right) \stackrel{\mathrm{D}}{\rightarrow} N\left(0, \eta^{2}\right),
$$


where $N(\cdot, \cdot)$ denotes the normal distribution, $\stackrel{\mathrm{D}}{\rightarrow}$ denotes convergence in distribution, and

$$
\eta^{2}= \begin{cases}\frac{1}{3} & \text { if } \mu=0, \\ \frac{\mathrm{e}^{-2 \mu b}\left(\mathrm{e}^{2 \mu b}-\mathrm{e}^{-2 \mu b}-4 \mu b\right)}{\left(1-\mathrm{e}^{-2 \mu b}\right)^{3}} & \text { if } \mu \neq 0 .\end{cases}
$$

Proof. With $f$ as in the proof of Theorem 4, (12) yields

$$
\sqrt{[M, M](t)}\left(\frac{U(t)}{[M, M](t)}-c\right)=\frac{(f(0)-f(V(t)))}{\sqrt{[M, M](t)}}+\frac{\int_{0}^{t} f^{\prime}(V(s)) \mathrm{d} M(s)}{\sqrt{[M, M](t)}} .
$$

We note that as $t \rightarrow \infty,(f(0)-f(V(t))) / \sqrt{[M, M](t)} \rightarrow 0$ a.s. Thus, what we need to show is that

$$
Y(t)=\frac{1}{\sqrt{[M, M](t)}} \int_{0}^{t} f^{\prime}(V(s)) \mathrm{d} M(s) \stackrel{\mathrm{D}}{\rightarrow} N\left(0, \eta^{2}\right) \quad \text { as } t \rightarrow \infty
$$

with $\eta^{2}$ as in (16). Now, $Y_{t}(s)=\int_{0}^{s} f^{\prime}(V(u)) \mathrm{d} M(u) / \sqrt{[M, M](t)}$ is a continuous martingale with quadratic variation $\left[Y_{t}, Y_{t}\right](s)$ given by $\int_{0}^{s}\left(f^{\prime}(V(u))\right)^{2} \mathrm{~d}[M, M](u) /[M, M](t)$ (we are considering a family $Y_{t}$ of martingales indexed by $t$ ). We make the simple but important observation that along the time-index 'diagonal', $Y$ and $Y_{t}$ coincide, i.e. $Y(t)=Y_{t}(t)$. It follows, by the martingale central limit theorem (see [9, pp. 338-340]) that $Y_{t}(t) \stackrel{\mathrm{D}}{\rightarrow} N\left(0, \eta^{2}\right)$ as $t \rightarrow \infty$, where $\eta^{2}=\lim _{t \rightarrow \infty}\left[Y_{t}, Y_{t}\right](t)$. What remains is thus to calculate

$$
\lim _{t \rightarrow \infty}\left[Y_{t}, Y_{t}\right](t)=\lim _{t \rightarrow \infty} \frac{\int_{0}^{t}\left(f^{\prime}(V(u))\right)^{2} \mathrm{~d}[M, M](u)}{[M, M](t)} .
$$

Recall that for $\mu=0$, we have $f^{\prime}(x)=x / b$ and for $\mu \neq 0, f^{\prime}(x)=\left(1-\mathrm{e}^{-2 \mu x}\right) /\left(1-\mathrm{e}^{-2 \mu b}\right)$. Take $r(x)=x^{2} / b^{2}$ for $\mu=0$ and $r(x)=\left(\left(1-\mathrm{e}^{-2 \mu x}\right) /\left(1-\mathrm{e}^{-2 \mu b}\right)\right)^{2}$ for $\mu \neq 0$. From Table 1 , we can find solutions to $(\mathscr{L} f)(x)=K+r(x)$. If we require that $f^{\prime}(0)=f^{\prime}(b)=0$ it turns out that $K=-\frac{1}{3}$ if $\mu=0$ and

$$
K=\frac{\left(-1-\mathrm{e}^{-2 \mu b}+4 \mu b \mathrm{e}^{-2 \mu b} /\left(1-\mathrm{e}^{-2 \mu b}\right)\right)}{\left(1-\mathrm{e}^{-2 \mu b}\right)^{2}}=\frac{\mathrm{e}^{-2 \mu b}\left(-\mathrm{e}^{2 \mu b}+\mathrm{e}^{-2 \mu b}+4 \mu b\right)}{\left(1-\mathrm{e}^{-2 \mu b}\right)^{3}} \quad \text { if } \mu \neq 0 .
$$

Equation (16) now follows from Lemma 1.

Remark 2. The condition in Theorem 5 that $[M, M]$ is deterministic looks strict. However, it is crucial that the processes $Y_{t}$ defined in the proof are local martingales; see [9]. Suppose that $M$ is a local martingale. Then $\int_{0}^{s} f^{\prime}(V(u)) \mathrm{d} M(u)$ is a local martingale, but it may be that $Y_{t}$ is not. The problem is that $[M, M](t)^{-1 / 2}$ need not be measurable with respect to $\mathscr{F}(s), s<t$. It is not obvious how to expand the filtration to make $Y_{t}$ measurable and a local martingale at the same time.

Remark 3. The reader may wonder why we do not take (6) as a starting point in the proofs of Theorems 4 and 5. We could actually derive the law of large numbers (Theorem 4) by using (6) to identify $U$ and Lemma 1 to evaluate $\lim _{t \rightarrow \infty} \int_{0}^{t} V(s) \mathrm{d}[M, M](s) /[M, M](t)$. However, the problem is that when we then try to prove Theorem 5 we can conclude that as $t \rightarrow \infty, U$ fulfills the condition

$$
2 b U(t)=-V(t)^{2}+2 \int_{0}^{t} V(s) \mathrm{d} M(s)+2 b K[M, M](t)+\mathrm{o}([M, M](t)),
$$


where $\mathrm{o}([M, M](t)) /[M, M](t) \rightarrow 0$, rather than the stronger condition

$$
U(t)=f(0)-f(V(t))+\int_{0}^{t} f^{\prime}(V(s)) \mathrm{d} M(s)+K[M, M](t),
$$

which follows by finding a solution to $(\mathscr{L} f)(x)=K$ satisfying $f^{\prime}(0)=0$ and $f^{\prime}(b)=1$, and applying (12). Since $o([M, M](t)) / \sqrt{[M, M](t)}$ need not go to 0 , we cannot control $\sqrt{[M, M](t)}(U(t) /[M, M](t)-K)$ in (17) and the method of proof that we use will not work.

The following approach to the derivation of Theorem 4 and Theorem 5 as well as the idea of how to weaken the assumptions in Theorem 5 was suggested to us by the anonymous referee. Besides being elegant it provides a nice explanation of why the constants $\eta^{2}$ and $c$ appearing in Theorem 4 and Theorem 5 are the same as in their counterpart in [22, Proposition 1], which is valid for Brownian motion. In (3), we use the representation $M(t)=B([M, M](t))$ a.s. where $B$ is the Dambis-Dubins-Schwarz Brownian motion of $M$; see, e.g. [19, Theorem 1.6]. Then, we obtain

$$
V(t)=V(0)+B([M, M](t))+\mu[M, M](t)+L(t)-U(t) .
$$

Now, if we take $X(t)=B(t)+\mu t$ and replace $t$ by $[M, M](t)$ in (3), we obtain

$$
\begin{aligned}
& V^{\mathrm{B}}([M, M](t))= \\
& V(0)+B([M, M](t))+\mu[M, M](t)+L^{\mathrm{B}}([M, M](t))-U^{\mathrm{B}}([M, M](t)),
\end{aligned}
$$

where $\left(V^{\mathrm{B}}, L^{\mathrm{B}}, U^{\mathrm{B}}\right)$ is the solution to the Skorokhod problem when the input is Brownian motion with drift. By comparing (18) and (19) and applying the uniqueness result for $(V, L, U)$ discussed in [17], we conclude that a.s. $U(t)=U^{\mathrm{B}}([M, M](t))$. Since we assume that $[M, M](t) \rightarrow \infty$ a.s. as $t \rightarrow \infty$, Theorem 4 and Theorem 5 follow easily from [22, Proposition 1], which tells us that

$$
\frac{U^{\mathrm{B}}(t)}{t} \rightarrow c \quad \text { a.s., } \quad \frac{\left(U^{\mathrm{B}}(t)-c t\right)}{\sqrt{t}} \stackrel{\mathrm{D}}{\rightarrow} N\left(0, \eta^{2}\right) \quad \text { as } t \rightarrow \infty .
$$

Let $\mathbb{Q}$ denote the rational numbers and let $Y_{q}=\left(U^{\mathrm{B}}(q)-c q\right) / \sqrt{q}, q \in \mathbb{Q}$. It follows follows by (20) that

$$
Y_{q} \stackrel{\mathrm{D}}{\rightarrow} N\left(0, \eta^{2}\right) \quad \text { as } \mathbb{Q} \ni q \rightarrow \infty .
$$

A general version of the Anscombe theorem (see, e.g. [4]) allows us to conclude that

$$
Y_{\tau(t)} \stackrel{\mathrm{D}}{\rightarrow} N\left(0, \eta^{2}\right) \quad \text { as } t \rightarrow \infty,
$$

for a stochastic index process $\tau(t)$, provided that $\tau(t) \in \mathbb{Q}$,

$$
\frac{\tau(t)}{b(t)} \stackrel{\mathbb{P}}{\rightarrow} 1 \text { as } t \rightarrow \infty,
$$

for some sequence of positive real numbers $\{b(t), t \geq 0\}$ where $b(t) \uparrow \infty$ and that given $\epsilon>0$ and $\phi>0$, there exist $\delta>0$ and $q_{0}$, such that, for all $q>q_{0}$,

$$
\mathbb{P}\left(\max _{\{r:|r-q|<q \delta\}}\left|Y_{r}-Y_{q}\right|>\epsilon\right)<\phi .
$$


Let us choose a continuous local martingale $M$ with a nondeterministic $[M, M]$ and take $\tau(t)$ to be a suitable rational approximation of $[M, M](t)$. If we manage to verify (21) and (22), the above should be adequate to show that Theorem 5 actually holds for this particular choice of $M$. However, to the best of the author's knowledge, (21) and (22) are not automatically satisfied for $M$ with a general nondeterministic $[M, M]$, which means that the verification must be made on a case-by-case basis.

We will conclude the paper by having a look at the asymptotic behavior of the 'time-average' of the reflected process $V$. Let $Y(t, \alpha)=\int_{0}^{t} \mathrm{e}^{\alpha V(s)} \mathrm{d}[M, M](s)$.

Theorem 6. As $t \rightarrow \infty, Y(t, \alpha) /[M, M](t) \rightarrow d$ a.s., where

$$
d= \begin{cases}\frac{\left(\mathrm{e}^{\alpha b}-1\right)}{\alpha b} & \text { if } \mu=0, \alpha \neq 0, \\ \frac{\left(\mu \mathrm{e}^{\alpha b+2 \mu b}-\mu\right)}{\left((\mu+\alpha / 2)\left(\mathrm{e}^{2 \mu b}-1\right)\right)} & \text { if } \mu \neq 0, \alpha \neq-2 \mu .\end{cases}
$$

Proof. We consult Table 1 to find solutions of $(\mathcal{L} f)(x)=K+\mathrm{e}^{\alpha x}$. Then we choose $K$ and $K_{1}$ such that $f^{\prime}(0)=f^{\prime}(b)=0$. The part of (23) to the right of the ' $\{$ ' shows $-K$. We apply Lemma 1 to finish the proof.

We recognize $d=d(\alpha)$ in (23) as the moment generating function of a uniform distribution on $[0, b]$ when $\mu=0$ and a truncated exponential distribution with density $2 \mu \mathrm{e}^{2 \mu x} /\left(\mathrm{e}^{2 \mu b}-\right.$ $1), 0 \leq x \leq b$, when $\mu \neq 0$. The following example shows an application of Theorem 6 .

Example 1. Suppose that we take $M(t)=\sigma B(t)$, so that $X(t)=\sigma B(t)+\mu t$, i.e. $X$ is Brownian motion with drift and $[M, M](t)=\sigma^{2} t$. This example was treated in [22]. It was proven in [17] that the $V$ corresponding to this input is regenerative with nonlattice cycle length distribution with finite mean. Now, according to Theorem 6, it holds that

$$
\frac{1}{t} \int_{0}^{t} \mathrm{e}^{\alpha V(s)} \mathrm{d} s \rightarrow d \quad \text { a.s. as } t \rightarrow \infty,
$$

and since $V$ is regenerative, it follows by [5, Theorems 1.2 and 3.1] that there exists a limiting (thus stationary) distribution $\pi$ of $V$, specified by $\int_{0}^{b} \mathrm{e}^{\alpha x} \pi(\mathrm{d} x)=d$, and from (23) we conclude that $\pi$ corresponds to a uniform distribution on $[0, b]$ when $\mu=0$ and a truncated exponential distribution when $\mu \neq 0$. We have thus derived the stationary distribution of the process resulting from a two-sided reflection of Brownian motion with drift without performing optional stopping of the Wald martingale (or the Kella-Whitt martingale), which is the typical way of approaching this problem; see, e.g. [5, Example 3.6] and [6].

On the other hand, since $V$ is regenerative and $[M, M](t)=\sigma^{2} t$, we may conclude directly from results for regenerative processes (this is precisely what the authors do in [22]) that

$$
\lim _{t \rightarrow \infty} \frac{\int_{0}^{t} r(V(s)) \mathrm{d}[M, M](s)}{[M, M](t)}=\lim _{t \rightarrow \infty} \frac{\int_{0}^{t} r(V(s)) \mathrm{d} s}{t}=\int_{0}^{b} r(x) \pi(\mathrm{d} x),
$$

and since $\pi$ can be found in the literature, there is no need for Lemma 1 and finding the potentially messy solutions to $(\mathcal{L} f)(x)=K+r(x)$, where $r(x) \neq 0$.

Theorem 7. Let $d$ be as in (23) and $M$ be a martingale with deterministic quadratic variation. As $t \rightarrow \infty$,

$$
\sqrt{[M, M](t)}\left(\frac{Y(t, \alpha)}{[M, M](t)}-d\right) \stackrel{\mathrm{D}}{\rightarrow} N\left(0, \eta^{2}\right)
$$


where

$$
\eta^{2}=\frac{4 d^{2} b^{2}}{3}+\frac{4\left(\mathrm{e}^{-\alpha b}-\mathrm{e}^{\alpha b}+2 \alpha b\right)}{\left(\alpha^{2}\left(1-\mathrm{e}^{\alpha b}\right)\right)}+\frac{4 d\left(2\left(\mathrm{e}^{\alpha b}-1\right) / \alpha^{2}-2 b \mathrm{e}^{\alpha b} / \alpha+b^{2}\right)}{\alpha b}
$$

if $\mu=0$ and $\alpha \neq 0$, whereas if $\mu \neq 0$ and $\alpha \neq-2 \mu$ and $\alpha \neq 0$, then

$$
\begin{aligned}
\eta^{2}= & \frac{d^{2} \mathrm{e}^{-2 \mu b}\left(\mathrm{e}^{2 \mu b}-\mathrm{e}^{-2 \mu b}-4 \mu b\right)}{\left(\mu^{2}\left(1-\mathrm{e}^{-2 \mu b}\right)\right)}+\frac{4 \mathrm{e}^{\alpha b}\left(\mathrm{e}^{-\alpha b}-\mathrm{e}^{\alpha b}+2 \alpha b\right)}{\left((\alpha+2 \mu)^{2}\left(1-\mathrm{e}^{\alpha b}\right)\right)} \\
+ & 4 d\left[1-\frac{\mu(2 \mu+2 b \alpha(\alpha+2 \mu))}{(\alpha(\alpha+2 \mu))}-\frac{2 \mu \mathrm{e}^{\alpha b}}{(\alpha+2 \mu)}+\frac{2 \mu \mathrm{e}^{(\alpha-2 \mu) b}}{\alpha}\right] \\
& \times\left[\left(1-\mathrm{e}^{-2 \mu b}\right) \mu(\alpha+2 \mu)\right]^{-1} .
\end{aligned}
$$

Proof. The proof is identical to the proof of Theorem 5, except that $\eta^{2}$ is of a different form. We have that the solution to $(\mathcal{L} f)(x)=K+\mathrm{e}^{\alpha x}$ satisfying $f^{\prime}(0)=f^{\prime}(b)=0$ is given by

$$
f^{\prime}(x)= \begin{cases}-2 \mathrm{~d} x+\frac{2\left(\mathrm{e}^{\alpha x}-1\right)}{\alpha} & \text { if } \mu=0, \\ \frac{-d\left(1-\mathrm{e}^{-2 \mu x}\right)}{\mu}-\frac{2\left(1-\mathrm{e}^{\alpha x}\right)}{(\alpha+2 \mu)} & \text { if } \mu \neq 0 .\end{cases}
$$

We take $r(x)=f^{\prime}(x)^{2}$ and solve $(\mathcal{L} f)(x)=K+r(x)$ by consulting Table 1 . The details consist of tedious but straightforward algebra and are omitted. We finish the proof by applying Lemma 1.

It is one of the main goals of the theory of regenerative processes to state and prove equivalents of Theorems 6 and 7; see [5, pp. 177-179]. There are, however, some noteworthy differences between this paper and the classical approach for regenerative processes. First, in regenerative process theory, one typically expresses the global time-average $\lim _{t \rightarrow \infty} t^{-1} \int_{0}^{t} r(V(s)) \mathrm{d} s$ via an average formed over a cycle, i.e. one goes from local cycle properties to a global result. Here, we can only say something about the global average (not very surprising since we have no cycles in general). Secondly, the results in [5] are formulated for general $r$, i.e. the only requirement is that integration makes sense. This is in contrast to Theorem 6 , where we take $r(x)=\mathrm{e}^{\alpha x}$. We go from a general $r$ to an $r$ belonging to this parametric family for two reasons. First, we can compute only exact averages for $r$ such that we can find an explicit solution to $(\mathscr{L} f)(x)=K+r(x)$ satisfying $f^{\prime}(0)=f^{\prime}(b)=0$. Secondly, we can rather easily generalize the results shown for this particular choice of $r$. For example, suppose that we take $g$ continuous such that $g(0)=g(b)$. It follows by Weirstrass' theorem that $g$ can be approximated by a trigonometric polynomial in the sense that for each $\varepsilon>0$, there exists a polynomial $p$ such that $\sup _{0 \leq x \leq b}\left|g(x)-p\left(\mathrm{e}^{i 2 \pi x / b}\right)\right|<\varepsilon$. Then clearly,

$$
\left|\frac{\left(\int_{0}^{t} g(V(s)) \mathrm{d}[M, M](s)-\int_{0}^{t} p\left(\mathrm{e}^{i 2 \pi V(s) / b}\right) \mathrm{d}[M, M](s)\right)}{[M, M](t)}\right| \leq \varepsilon,
$$

and we may approximate $\lim _{t \rightarrow \infty} \int_{0}^{t} g(V(s)) \mathrm{d}[M, M](s) /[M, M](t)$ arbitrarily well by the corresponding limit for a trigonometric polynomial which we can compute using (23) (and the linearity of integration). The condition $g(0)=g(b)$ is no restriction. To see this, note that a general continuous $g$ can be written as

$$
g(x)=\left(\frac{g(x)-x(g(b)-g(0))}{b}\right)+\frac{x(g(b)-g(0))}{b},
$$


so that

$$
\begin{aligned}
\lim _{t \rightarrow \infty} \frac{\int_{0}^{t} g(V(s)) \mathrm{d}[M, M](s)}{[M, M](t)}= & \lim _{t \rightarrow \infty} \frac{\int_{0}^{t}(g(V(s))-V(s)(g(b)-g(0)) / b) \mathrm{d}[M, M](s)}{[M, M](t)} \\
& +\lim _{t \rightarrow \infty} \frac{\int_{0}^{t} V(s)(g(b)-g(0)) / b \mathrm{~d}[M, M](s)}{[M, M](t)}
\end{aligned}
$$

The first part in the right-hand side of (24) is a continuous function whose values at the endpoints coincide. Furthermore, we can easily compute the rightmost global time-average in (25) using Lemma 1 and Table 1.

\section{Acknowledgement}

The author wishes to thank an anonymous referee for suggesting the alternative proofs of Theorems 4 and 5 (based on the representation of a continuous local martingale as time-changed Brownian motion) as well as for other useful remarks that greatly improved the quality of this paper.

\section{References}

[1] Andersen, L. N. (2011). Subexponential loss rate asymptotics for Lévy processes. Math. Meth. Operat. Res. 1, 91-108.

[2] ANDERSEN, L. N. AND ASMUSSEN, S. (2011). Local time asymptotics for centered Lévy processes with two-sided reflection. Stoch. Models 27, 202-219.

[3] ANDERSEN, L. N. AND MANDJES, M. (2009). Structural properties of reflected Lévy processes. Queueing Systems 63, 301-322.

[4] Anscombe, F. J. (1952). Large-sample theory of sequential estimation. Proc. Camb. Phil. Soc. 48, 600-607.

[5] Asmussen, S. (2003). Applied Probability and Queues, 2nd edn. Springer, New York.

[6] Asmussen, S. AND Pihlsgård, M. (2007). Loss rates for Lévy processes with two reflecting barriers. Math. Operat. Res. 32, 308-321.

[7] CoOper, W. L., Schmidt, V. ANd SERfozo, R. F. (2001). Skorohod-Loynes characterizations of queueing, fluid, and inventory processes. Queueing Systems 37, 233-257.

[8] D'Auria, B. AND Kella, O. (2012). Markov modulation of a two-sided reflected Brownian motion with application to fluid queues. Stoch. Process. Appl. 122, 1566-1581.

[9] Ethier, S. N. AND Kurtz, T. G. (1986). Markov Processes: Characterization and Convergence. John Wiley, New York.

[10] Friedman, A. (1982). Foundations of Modern Analysis. Dover, New York.

[11] Kella, O. (2006). Reflecting thoughts. Statist. Prob. Lett. 76, 1808-1811.

[12] Kella, O., Boxma, O. And Mandjes, M. (2006). A Lévy process reflected at a Poisson age process. J. Appl. Prob. 43, 221-230.

[13] KIm, H. S. AND SHROFF, N. B. (2001). On the asymptotic relationship between the overflow probability and the loss ratio. Adv. Appl. Prob. 33, 836-863.

[14] Kruk, L., Lehoczky, J., Ramanan, K. AND Shreve, S. (2007). An explicit formula for the Skorokhod map on [0, a]. Ann. Prob. 35, 1740-1768.

[15] PALMOWSKI, Z. AND ŚWIA̧TEK, P. (2011). Loss rate for a general Lévy process with downward periodic barrier. In New Frontiers in Applied Probability (J. Appl. Prob. Spec. Vol. 48A), Applied Probability Trust, Sheffield, pp. 99-108.

[16] PIHLsGÅRD, M. (2005). Loss rate asymptotics in a $G I / G / 1$ queue with finite buffer. Stoch. Models 21,913-931.

[17] PihlsGÅRD, M. AND GLYNN, P. W. (2013). On the dynamics of semimartingales with two reflecting barriers. $J$. Appl. Prob. 50, 671-685.

[18] Protter, P. E. (2004). Stochastic Integration and Differential Equations, 2nd edn. Springer, Berlin.

[19] Revuz, D. AND Yor, M. (1999). Continuous Martingales and Brownian Motion, 3rd edn. Springer, Berlin.

[20] Sato, K.-I. (1999). Lévy Processes and Infinitely Divisible Distributions. Cambridge University Press.

[21] Stadje, W. (1993). A new look at the Moran dam. J. Appl. Prob. 30, 489-495.

[22] Zhang, X. And GlynN, P. W. (2011). On the dynamics of a finite buffer queue conditioned on the amount of loss. Queueing Systems 67, 91-110. 